Cahiers
de a Recherche
Fourles Droits
Fondamentaux

Cahiers de la recherche sur les droits fondamentaux

5 | 2006

L'enfant

\title{
Les droits de l'enfant abandonné (1811-2003)
}

\author{
Ivan Jablonka
}

\section{OpenEdition}

Journals

Édition électronique

URL : https://journals.openedition.org/crdf/7127

DOI : $10.4000 /$ crdf. 7127

ISSN : 2264-1246

Éditeur

Presses universitaires de Caen

Édition imprimée

Date de publication : 31 décembre 2006

Pagination : 23-30

ISBN : 978-2-84133-277-9

ISSN : 1634-8842

Référence électronique

Ivan Jablonka, «Les droits de l'enfant abandonné (1811-2003) », Cahiers de la recherche sur les droits fondamentaux [En ligne], 5 | 2006, mis en ligne le 15 décembre 2020, consulté le 14 novembre 2022. URL : http://journals.openedition.org/crdf/7127 ; DOI : https://doi.org/10.4000/crdf.7127 


\title{
Les droits de l'enfant abandonné (1811-2003)
}

\author{
Ivan JABLONKA \\ Maître de conférences \\ Université du Maine - Le Mans
}

\author{
I. Recueillir et élever l'enfant abandonné \\ A. Des enfants trouvés aux "moralement abandonnés" \\ B. L'Assistance publique en charge des pupilles \\ II. L'enfant et ses origines \\ A. Connaître ses parents \\ B. De la rétention d'informations à l'ouverture des dossiers
}

Enfants rejetés, enfants en surnombre, enfants auxquels on impute toutes sortes de tares, les trouvés du XVII et du XVIII ${ }^{\mathrm{e}}$ siècle n'ont souvent connu que de mornes destins. En premier lieu, c'est la mort qui les attendait. S'ils survivaient aux terribles conditions du transport et de la mise en nourrice, les enfants abandonnés devenaient généralement artisans, valets de ferme, servantes, soldats ou matelots. Le hasard, sous l'Ancien Régime et dans la première moitié du XIX ${ }^{\mathrm{e}}$ siècle, entre peu dans le cours de ces existences : tout semble joué d'avance.

Au XIX ${ }^{\mathrm{e}}$ siècle, les écrivains ont mis en scène la souffrance d'individus brisés, promis au vagabondage et à la misère après une enfance sans joie. À partir du milieu du XIX ${ }^{\mathrm{e}}$ siècle, c'est le tort fait à tous les enfants qui est dénoncé, et La Petite Fadette (1849), Le Petit Chose (1868) ou L'Enfant de Jules Vallès (1879) offrent l'image poignante d'êtres brisés, avilis, accablés par le surmenage et la pauvreté $^{1}$; mais la peine et l'humiliation, avant la rédemption finale, semblent d'abord réservées aux enfants sans famille. Dans le dernier tiers du siècle, les avanies subies par Simon, le petit bâtard outragé par ses camarades dans une nouvelle de Maupassant, par Rémy, le jeune vagabond de Sans famille, par Gavroche, Cosette et Gwynplaine, immortels personnages de la geste hugolienne, soulèvent la révolte et l'émoi des lecteurs français. Victimes d'un ordre social qui les rejette, ces enfants sans enfance, privés de racines et d'affection, endurent sans sourciller la faim, le froid, la solitude, l'exploitation, les accidents, le cynisme des hommes et les coups du sort : leur destin est de vivre à l'écart de la société honnête, loin des foyers où règne le bonheur.

Il semble, dès lors, que l'enfant abandonné soit par nature un être dépourvu de dignité et de droits. On pourrait être réticent à séparer le sort des pupilles de celui des enfants en général, tant il est vrai que ces derniers, au $\mathrm{XIX}^{\mathrm{e}}$ siècle, jouissent rarement d'un quelconque privilège attaché à leur âge : enfants pauvres, enfants dressés, enfants coupables, exploités au fond des mines, derrière les métiers à tisser, sur les navires ou dans les prisons, ils sont privés de tout droit ${ }^{2}$. Pourtant, sous Napoléon $\mathrm{I}^{\mathrm{er}}$

1. Voir M. Bethlenfalvay, Les Visages de l'enfant dans la littérature française du XIX siècle, Genève, Droz, 1979; G. Tison, Une mosä̈que d'enfants. L'enfant et l'adolescent dans le roman français (1876-189o), Arras, Artois Presses Université (Études littéraires et linguistiques), 1998.

2. Il faut attendre 1924 pour que la SDN rédige la première Déclaration des droits de l'enfant. L'ONU en adopte une deuxième en 1959. Sur le sujet, voir C. Rollet, Les Enfants au XIX siècle, Paris, Hachette Littératures, 2001, chapitre VII. 
comme sous l'ère républicaine, des facteurs objectifs prouvent l'infériorité des enfants abandonnés par rapport aux autres. En 1828, c'est sur des enfants trouvés que le docteur Billard effectue ses autopsies ${ }^{3}$, et c'est eux également que les spéculateurs veulent employer pour coloniser les déserts algériens ${ }^{4}$. Bien que d'inégale portée, ces deux expériences montrent que le corps des enfants abandonnés est moins précieux que celui des enfants de famille.

Doit-on en conclure que, au XIX ${ }^{\mathrm{e}}$ siècle et jusqu’à la fin de la Troisième République, les pupilles de l'Assistance publique vivent en dehors de la communauté, maintenus aux marges de la civilisation par le mépris et le soupçon, relégués dans le monde interlope de la dangerosité, persécutés comme des boucs émissaires? Pourtant, au sein de l'Assistance publique, certains droits ont été proclamés au bénéfice des enfants. Si cette administration promeut certains droits, à l'inverse, elle en refuse d'autres.

\section{Recueillir et élever l'enfant abandonné}

L'Assistance publique reconnaît un droit à tout enfant privé de soutien familial : celui d'être recueilli, entretenu et éduqué. Avant même l'édification de ces "masses de granit ${ }^{5}$ que constituent les grandes lois sociales votées sous la Troisième République, la France se dote d'un arsenal législatif et réglementaire permettant de prendre en charge les enfants sans parents.

\section{A. Des enfants trouvés aux « moralement abandonnés »}

Le décret du 19 janvier 1811 prévoit trois catégories d'enfants recueillis par les hospices : les trouvés, les abandonnés et les orphelins pauvres. L'enfant trouvé a été exposé dans un lieu public - escalier, porche, église, chambre d'hôtel, chemin creux - ou dans une tour; il est de père et de mère inconnus et l'identité de l'adulte qui a présidé à son abandon reste ignorée. Les enfants abandonnés sont nés de parents connus, mais ils « en sont délaissés, sans qu'on sache ce que les père et mère sont devenus, ou sans qu'on puisse recourir à eux ». L'orphelin pauvre est admis à l'Assistance publique parce qu'il reste sans ressources après le décès de ses père et mère ou tuteurs. Pendant et après la Première Guerre mondiale, les enfants dont le père est tombé au champ d'honneur sont des orphelins dits « de guerre ». Dans certains cas, ils deviennent pupilles de la nation; mais en aucun cas ils ne dépendent de l'Assistance publique. De manière générale, le secteur privé - par exemple les philanthropes et les orphelinats religieux - éprouve une prédilection marquée pour les orphelins pauvres, les «enfants du péché» (trouvés et abandonnés) étant laissés à la discrétion de l'Assistance publique.
À ces trois familles de base - trouvé, abandonné, orphelin - s'ajoute en 1889 une quatrième, celle des enfants dits «moralement abandonnés ». Dans la majeure partie du XIX ${ }^{\mathrm{e}}$ siècle, l'administration ne prévoit rien pour l'accueil des adolescents, puisqu'une circulaire ministérielle de 1823 refuse l'accès au service des enfants âgés de plus de 12 ans. À partir de 1838, l'Hospice des enfants trouvés reçoit bien quelques grands enfants, mais la plupart des adolescents sans famille sont voués à vagabonder. Pour pallier cette lacune, le Conseil général de la Seine étudie le 6 décembre 1879 un projet visant à admettre à l'Assistance publique de la Seine des enfants de plus de 12 ans vivant sans abri ni secours. Adopté à la session de novembre 1880 , le projet conduit à la création du service des moralement abandonnés à partir du $1^{\mathrm{er}}$ janvier 1881 , en dehors de tout cadre légal. Ce service accueille les petits vagabonds de 12 à 16 ans, les enfants maltraités par leurs parents ainsi que les mineurs acquittés selon l'article 66 du Code pénal.

L'expérience est assez probante pour que la notion d'«abandon moral» soit étendue au pays tout entier. Préparée par la loi Tallon de 1874 relative aux enfants de forains, la loi sur les enfants moralement abandonnés est votée le 24 juillet 1889 . Elle prévoit de déchoir de leurs droits les parents d'enfants négligés, maltraités ou insoumis, et de confier ces derniers à l'Assistance publique; selon l'article 17 du titre II, les parents peuvent également « déléguer » leurs droits de puissance paternelle à l'administration. Le texte de 1889 est ambigu. Tout en protégeant l'enfance malheureuse (et notamment les enfants martyrs victimes de parents indignes), il s'attache à lutter contre la délinquance juvénile dans les milieux populaires et à rééduquer les petits vagabonds. En brouillant les frontières entre enfance coupable et enfance victime, il renouvelle le préjugé selon lequel les enfants sans famille représentent peu ou prou un danger pour la société. La loi de 1889 confirme explicitement l'Assistance publique dans sa mission éducative : non seulement elle doit sauver de la mort les enfants trouvés et abandonnés, les pourvoir d'un placement et superviser leur éducation, mais elle est désormais chargée de rééduquer les moralement abandonnés, «ces orphelins dont les parents sont vivants» (selon l'expression de Jules Simon), en leur montrant le droit chemin en lieu et place d'une famille défaillante.

La loi du 19 avril 1898 sur la répression des violences et actes de cruauté envers les enfants participe du même mouvement: en prenant en charge à la fois les enfants «victimes et auteurs» d'infractions pénales, elle accentue le rapprochement entre enfance coupable et enfance malheureuse. Tout en aggravant certaines peines, elle donne davantage de raisons à l'État d'intervenir contre l'autorité du père. Une nouvelle catégorie est créée qui relève encore de l'Assistance publique : celle des enfants «en

3. C.-M. Billard, Traité des maladies des enfants nouveau-nés et à la mamelle, fondé sur de nouvelles observations cliniques et d'anatomie pathologique faites à l'hôpital des Enfants-trouvés de Paris, Paris, Baillière, 1828.

4. E. de Tocqueville, Des enfants trouvés et des orphelins pauvres comme moyen de colonisation de l'Algérie, Paris, Amyot, 1850 ; abbé Dubeau, L'Enfant trouvé et l'Algérie, ou colonisation agricole de l'Afrique française, Paris, chez Dubeau, 1860 ; L. Boutard, Colonisation en Algérie par les enfants assistés de France, Paris, Imprimerie de Veuve Walder, 1876.

5. C. Rollet, La Politique à l'égard de la petite enfance sous la Troisième République (1865-1939), Paris, INED, 1990, p. 131 sq. 
garde ». La diversification des modes d'assistance reflète le pouvoir que l'administration a gagné au détriment des familles et notamment des pères. De simple réceptacle habilité à recevoir le trop-plein des nouveau-nés indésirables, l'Assistance publique est devenue une instance éducative prête à se substituer aux familles incapables d'élever un enfant par elles-mêmes. Il serait pourtant erroné d'affirmer que l'État entend détruire l'influence de la famille, puisque la majorité des enfants assistés est placée non dans des établissements d'éducation collective mais en famille d'accueil : ce n'est donc pas l'institution familiale qui est remise en cause à la fin du XIX ${ }^{\mathrm{e}}$ siècle, mais l'éducation donnée au sein des classes populaires et la prégnance de la filiation biologique. Selon les lois de 1889 et de 1898 , un enfant peut être élevé par une autre famille si son intérêt y gagne: le lien du sang s'efface devant l'intérêt de l'enfant, ce qui prépare la législation de l'adoption de mineurs mise en œuvre à partir des années 1920.

Les diverses catégories d'enfants assistés utilisées au XIX siècle sont synthétisées dans la loi du 27 juin 1904. Ce texte fondamental répartit les enfants assistés en plusieurs populations. Les enfants trouvés ( $T)$, les enfants abandonnés (A), les orphelins $(\mathrm{O})$ et les enfants moralement abandonnés (MA) sont placés «sous la tutelle de l'autorité publique » et deviennent des «pupilles de l'Assistance publique» ou "pupilles de l'État». Pour une raison ou pour une autre, l'État s'est substitué à leurs géniteurs; il exerce la tutelle légale jusqu’à la majorité du pupille. Le lien du sang étant rompu, ces mineurs seront adoptables au regard des lois votées dans l'entre-deux-guerres.

Les enfants en dépôt, les enfants temporairement recueillis et les enfants en garde sont quant à eux placés "sous la protection publique». Ils dépendent toujours légalement de leurs parents et leur séjour à l'Assistance publique est provisoire ${ }^{6}$. Les enfants en dépôt (D) sont recueillis temporairement par le service des enfants assistés en raison de l'hospitalisation, de la détention, de la condamnation ou de l'internement de leurs parents. Depuis les années 1904-1906, les enfants admis par mesure de bienfaisance après demande de la famille et enquête de l'administration sont dits «temporairement recueillis » $(\mathrm{TR})^{7}$. Enfin, la loi de 1898 distingue les « enfants en garde victimes de délits » $(\mathrm{GV})$ et les « enfants en garde auteurs de délits » (GA).

Toutes ces catégories relèvent du service des enfants assistés, comme un spécialiste l'explique en 1912 : «L'expression générique d'enfants assistés s'applique aux pupilles de l'Assistance publique et aux enfants qui leur sont assimilés : enfants en garde et enfants en dépôt. Le caractère commun de tous ces enfants est de bénéficier de l'éducation donnée par le service des enfants assistés ${ }^{8}$. En définitive, le service des enfants assistés comprend les enfants en dépôt, les enfants temporairement recueillis, les enfants en garde et les pupilles de l'Assistance publique (ou pupilles de l'État).

\section{B. L'Assistance publique en charge des pupilles}

Après avoir inscrit, immatriculé et catégorisé l'enfant, l'administration ouvre un dossier à son nom. L'ancêtre du dossier est le livret où sont consignées plusieurs informations ayant trait à l'enfant. Sous l'Ancien Régime, l'Hospice des enfants trouvés délivre à la nourrice une «bulle» où sont portés le nom du meneur, le nom de l'enfant, son sexe, son âge, son numéro, sa date de réception, la liste de ses vêtures et enfin le nom de la nourrice. Cette bulle sera remplacée plus tard par un livret. Sous la Troisième République, le dossier personnel de l'enfant assisté rassemble un certain nombre de pièces qu'on peut classer en trois ensembles : les pièces génériques (procès-verbal d'abandon, certificat de naissance, acte de baptême, fiche de visites trimestrielles, livret de pupille, décisions du conseil de famille à propos des récompenses, de la dot ou du mariage), la correspondance générale (lettres des parents, de la nourrice, de l'inspecteur, du directeur de l'Assistance publique, du pupille lui-même ou de ses amis) et les pièces contingentes, généralement témoins d'un événement malheureux (accident, délit, évasion, viol, décès prématuré).

Qu'on les désigne sous le nom de bulle, livret ou dossier, ces pièces administratives contiennent au sujet du mineur une information cohérente, durable et personnelle. Il s'agit, là encore, d'une entreprise d'enregistrement et de fichage. Ce «dossier jamais clos», symptôme d'une société disciplinaire, identifierait selon Michel Foucault l'enfant assisté à d'autres catégories d'individus à contrôler - soldats, malades, prisonniers, déportés ${ }^{9}$. Cette conception, juste à certains égards, néglige le fait que la création d'un dossier vise aussi à faire respecter les droits de la personne - l'individualisation de l'enfant au sein d'énormes effectifs, l'attribution d'un placement approprié, l'établissement de sa future retraite, etc.

Le dossier individuel fait partie des archives de l'administration, mais il constitue aussi les seules archives de l'enfant. En effet, contrairement aux enfants de famille, le pupille n'est pas en mesure de consulter les pièces, photos et souvenirs que son enfance a laissé surnager. Il s'établit donc une sorte d'homologie entre l'enfant assisté et le dossier, son «frère de papier ${ }^{10}$, qui explique une part de l'énorme investissement affectif que les démarches

\footnotetext{
6. Les enfants secourus, dont le statut est précisé par la loi du 22 avril 1905 et qui relèvent du service des enfants assistés de la Seine, ne sont pris en charge que financièrement, parce que leurs parents sont sans ressources. L'attribution de secours vise précisément à éviter l'abandon.

7. Le règlement du 24 octobre 1906, prévu par l'article 58 de la loi du 27 juin 1904, mentionne que les enfants moralement abandonnés de la Seine admis dans les années 1880 - donc hors du cadre légal - seront appelés "temporairement recueillis ». Cette catégorie se distingue de celle des enfants « recueillis temporairement» (RT) qui, selon les termes de la loi du 15 avril 1943, désigne les enfants en dépôt. Cette même loi supprime les TR.

8. É. Alcindor, Les Enfants assistés, Paris, Émile Paul, 1912, p. 61.

9. Voir M. Foucault, Surveiller et Punir. Naissance de la prison, Paris, Gallimard (Bibliothèque des histoires), 1975, p. 300 sq.

10. G. Cesbron, Chiens perdus sans collier, Paris, Robert Laffont, 1954, p. 14.
} 
administratives peuvent susciter chez les intéressés. Le dossier, dépositaire de l'intime, présente un intérêt exceptionnel pour l'historien, mais aussi - avant même que ce dernier ne surgisse pour le profaner - pour l'individu sans famille dont il a suivi pas à pas l'enfance et l'adolescence.

Dans les zones de placement, les inspecteurs départementaux et les directeurs d'agence ont vocation à défendre les intérêts du pupille. Avant tout, l'administration fait respecter le droit des pupilles. Elle garantit l'exécution de la loi et des contrats par les employeurs. Quand en 1910 une pupille se blesse dans une usine de tissage, le directeur de l'agence d'Abbeville (Somme) exige que le patron prenne en charge tous les soins. Comme ce dernier fait des difficultés, l'administration générale lui annonce par lettre que, s'il persiste à refuser de payer les frais de maladie et l'intégralité des gages de l'élève pendant l'interruption de travail, il sera attaqué en justice ${ }^{11}$. En outre, le directeur veille au respect du contrat de travail. Le 16 juin 1898, dans cette même agence, un patron envoie une lettre au directeur Fennebresque l'informant qu'il souhaite rendre son pupille avant la Saint-Jean, contrairement à ce que stipulait le contrat signé en mars. Le directeur accepte, mais il précise au patron qu'il perdra ses «arrhes si [le garçon] est loué moins de 150 francs ». Finalement, le pupille est réengagé par son patron jusqu'au mois de juin 1899 avec un salaire annuel de 150 francs $^{12}$. En septembre 1898 , Fennebresque note qu'un garçon de 16 ans « a reçu trois gifles de son patron (et cela sans motif). [Le patron] avait déjà donné un coup de pied à l'élève il y a environ dix jours. Avertissement donné au patron de ne plus recommencer, sinon je poursuis ». Trois ans plus tard, en novembre 1901, le pupille est renvoyé sans préavis par un autre patron. Fennebresque adresse alors l'élève au propriétaire avec une lettre rappelant son autorité et les termes du contrat ${ }^{13}$.

Ces marques de soutien ne sont jamais aussi éclatantes que lors des mariages: en cette occasion, les jeunes filles demandent au directeur de les conduire à l'autel ou chez le maire. En 1895, le directeur de l'agence de Romorantin (Loir-et-Cher) accompagne quatre pupilles à la mairie : "Nos enfants attachent toujours un grand prix à cette démonstration qu'ils considèrent comme un honneur ${ }^{14}$. En avril 1900, le futur d'une pupille invite le même directeur à la cérémonie ${ }^{15}$. En 1913, le directeur de l'agence d'Avallon (Yonne) se rend à Sainte-Magnance dans la matinée pour «assister au mariage» d'une pupille ${ }^{16}$. En juillet 1924, une jeune fille de l'agence de Doullens (Somme) écrit au directeur pour le convier «à la noce » ${ }^{17}$. La même année, une jeune fille de 22 ans annonce au directeur Demacon qu' elle se marie et ajoute: "Je serais très heureuse si vous acceptiez de m'accompagner pour la cérémonie et de me servir de père ». Dès le lendemain, Demacon lui attribue une dot de 400 francs ${ }^{18}$.

L'administration se charge aussi de défendre l'honneur des pupilles filles-mères délaissées par leur séducteur, en faisant pression sur lui et sa famille. Dans le cas où le séducteur fuit ses responsabilités, l'administration entre en jeu pour prêter assistance à la fille-mère. D'abord, la jeune mère a droit à des secours financiers. Ensuite, le directeur d'agence s'attelle à la défense de la pupille délaissée en essayant de faire pression sur le séducteur. Sa tâche est compliquée par l'article 340 du Code civil de 1804 qui interdit la recherche en paternité (sauf en cas d'enlèvement). Malgré ce contexte très défavorable, le directeur essaie d'aider la pupille. Généralement, il ne se contente pas d'écrire au garçon; il se déplace chez les parents, tente de ramener le jeune père à la raison et, en cas d'échec, négocie une indemnité. En 1910, le directeur de l'agence d'Avallon (Yonne) se rend dans un village pour obtenir des renseignements sur l'origine de la grossesse de la pupille W. Il relate son enquête :

«Je vais ensuite voir $\mathrm{M}^{\mathrm{me}}$ Veuve $\mathrm{B}$., mère du jeune homme accusé par l'élève W. de l'avoir séduite. Cette pauvre femme est navrée, et de son attitude semble ressortir que son fils n'en est pas à sa première sottise; elle envoie chercher le jeune homme et, en présence de sa mère et de sa sœur aînée, je le presse de questions. Il reste quelque peu interloqué. Ses yeux me prouvent qu'il est coupable, mais de sa bouche sort une dénégation intéressée. Pour essayer de le décider à un aveu, je lui laisse entendre que s'il ne me dit pas la vérité, c'est la Justice qui informera. Il ne répond pas, mais sa sœur et sa mère semblent plus inquiètes. Le jeune homme prétend que W. aurait écrit "au Dugna” pour se marier avec lui. Il y aura lieu de compléter ces divers renseignements avant d'adresser un rapport à l'administration ${ }^{19}$.

Après la fin de la période de tutelle, la bienveillance du directeur ne s'interrompt pas d'un coup et celui-ci maintient les liens avec les pupilles majeurs qui le souhaitent. Dans les années 1890, le directeur de l'agence d'Abbeville (Somme) organise des comités de patronage ${ }^{20}$. En outre, le directeur apporte son aide financière aux anciens pupilles nécessiteux - dans la mesure où l'administration juge

11. Archives de la Ville de Paris (AVP), EA 4450, Bérénice Maxon, née 25 mai 1893, admise 5 mars 1895 (à 2 ans), abandonnée, agence d'Abbeville. Les enfants relevant de l'Assistance publique de la Seine étaient transférés depuis Paris jusque dans des agences de placement disséminées sur tout le territoire français. Les enfants abandonnés dans les villes de province restaient sur place au sein de l'Assistance publique de leur département.

12. Direction de l'action sociale, de l'enfance et de la santé de la mairie de Paris (DASES), Marcel Caver, né 2 janvier 1882 , admis 17 octobre 1895 (à 13 ans et demi), orphelin, agence de Romorantin.

13. DASES, Désiré Pasquier, né 2 janvier 1882, admis 2 février 1882 (à 1 mois), abandonné, agence de Romorantin.

14. AVP, D1X ${ }^{4} 46$, agence de Romorantin, inspection de M. Thirault (novembre 1896), exercice 1895.

15. DASES, Mélanie Mesnil, née 11 janvier 1882, admise 2 février 1882 (à 3 semaines), abandonnée, agence de Romorantin.

16. AVP, journal de Mathieu Tamet, directeur de l'agence d'Avallon (9 septembre 1913).

17. DASES, Bernadette Godin, née 12 août 1902, admise 13 août 1902 (à 1 jour), agence de Doullens.

18. DASES, Ẽve Rutabeuf, née 21 septembre 1902, admise 4 octobre 1902 (à 2 semaines), agence de Doullens.

19. AVP, journal de Mathieu Tamet, directeur de l'agence d'Avallon (23 mars 1910).

20. AVP, D1X ${ }^{4} 46$, agence d'Abbeville, inspection de M. Villebrun (octobre 1896), exercice 1895. 
qu'ils l'ont méritée. Une fois majeur, Jean Genet implore les secours de l'Assistance publique et l'administration lui vient en aide à trois reprises en mars 1934, en avril 1936 et en mars 1939. La première fois, l'inspecteur départemental de l'Hérault informe le directeur général de l'Assistance publique à Paris que Genet s'est présenté à lui « sans travail et sans ressources. [...] Plus que sommairement vêtu, je lui ai procuré des vêtements convenables et assuré provisoirement sa subsistance $»^{21}$.

L'Assistance publique, soucieuse des intérêts financiers, moraux et affectifs du pupille, lui reconnaît donc un certain nombre de droits: être singularisé parmi des dizaines de milliers d'autres enfants, être suivi à l'aide d'un dossier mis à jour, être entretenu au sein d'une famille d'accueil, être titulaire d'un contrat de travail écrit, bénéficier lors des grandes occasions d'une représentation symbolique de la parenté, jouir d'une protection légale en cas d'agression et d'une forme de sécurité financière en cas de déveine. Outre le fait qu'elle reste parfois toute théorique, cette sollicitude a des limites: elle implique la rupture avec la famille biologique. Les droits consentis à l'enfant sans famille ont donc pour contrepartie l'interdiction de renouer avec le milieu d'origine.

\section{L'enfant et ses origines}

C'est Freud qui, le premier, a étudié la manière dont tout enfant se construit un «roman familial», projetant fantaisies et fantasmes sur ses parents biologiques. Dans le cas des enfants abandonnés, la séparation précoce, le secret des origines et l'aura de mystère dans lequel sont maintenus les parents tendent à compliquer à l'excès les linéaments du roman familial. Depuis 1945, psychanalystes, psychologues et pédopsychiatres insistent beaucoup sur les séquelles que conserve l'enfant privé de l'affection parentale (notamment maternelle). Or l'Assistance publique joue un rôle actif dans cette séparation.

\section{A. Connaître ses parents}

C’est généralement le service militaire qui révèle aux pupilles leur isolement familial. Même si les garçons de l'Assistance publique nouent quelques amitiés au régiment, ils ressentent une très vive solitude. Contrairement aux fils de famille, ils ne reçoivent aucune lettre. En 1923, exilé en Allemagne, un pupille avoue au directeur qu'il a « un peu le cafard "22. En 1929, à Meknès, un garçon demande qu'on lui trouve « une petite Marraine parmi les jeunes filles de l'Assistance publique. N'ayant plus aucun parent et étant pour faire ma carrière militaire, je serais heureux comme mes camarades de recevoir de temps à autres une petite missive qui m'enlèverait le cafard ${ }^{23}$. C'est à cette époque précise que les pupilles commencent à s'enquérir activement de leur famille biologique. En 1922, un soldat en garnison à Pithiviers demande des « renseignements concernant [ses parents] et principalement leur adresse actuelle que je serais très heureux de connaître ${ }^{24}$. En mai 1922, un garçon abandonné à l'âge de 4 mois demande à son " cher tuteur» des renseignements sur sa famille:

« [Je voudrais connaître] la situation de celle qui m’a donné le jour et savoir si elle existe encore, [pour que] je puisse lui faire souvenir qu'elle a un devoir à remplir en m'aidant à reprendre le droit chemin que j'avais perdu un instant par irréflexion : jamais encore je n'ai pu entendre parler de cet être que l'on doit bien chérir. [...]. Si je retrouve ma mère cela fera certainement mon bonheur, car j'aurai toujours quelqu'un pour me bien conseiller $»^{25}$.

Les filles ne se préoccupent pas de retrouver leurs parents avant l'âge de la majorité. Dès qu'elles ont 21 ans, elles s'adressent à l'administration de l'Assistance publique, d'elles-mêmes ou par l'intermédiaire de leur patron. En août 1923, au moment où son compte de tutelle est soldé, une pupille demande si elle a « encore [ses] parents et si c'est possible [d'avoir] leur nom ${ }^{26}$. En décembre 1924, une jeune femme de 21 ans et 2 mois, mariée à un ancien pupille de 23 ans journalier à Gièvres (Loir-et-Cher), demande au directeur de l'Assistance publique « quelques détails au sujet de $[\mathrm{sa}]$ mère si elle est vivante $[$ sic $] »$. Trois ans plus tard, elle réclame à nouveau «quelques petits renseignements au sujet de $[\mathrm{sa}]$ mère $[. .$.$] . Où est-elle?$ Que devient-elle? Ainsi que celle de mon mari mis à l'Assistance sous le nom de Charles ${ }^{27}$.

La plupart des enfants abandonnés essaient d'entrer en contact avec leurs parents biologiques, ou du moins de connaître leur identité. Les garçons, pragmatiques, posent la question quand ils en ressentent le besoin (généralement à l'époque du service militaire) tandis que les filles, plus soucieuses de respecter les formes, attendent l'âge de la majorité - ce qui ne signifie pas qu'elles n'y aient pas pensé avant. Ni le transfert à la campagne, ni l'accueil dans une famille autochtone, ni l'insertion professionnelle ne réussissent à chasser les parents de l'esprit des pupilles. Or, de même que l'administration tente de réduire à néant l'influence des familles biologiques, de même elle conserve soigneusement le secret des origines.

\section{B. De la rétention d'informations à l'ouverture des dossiers}

Tant que les nouveau-nés étaient exposés dans des tours (ce qui faisait d'eux, légalement, des enfants trouvés),

21. DASES, Jean Genet, né 19 décembre 1910, admis 28 juillet 1911 (à 7 mois), abandonné, agence de Saulieu.

22. DASES, Simon Gabelon, né 5 novembre 1902, admis 19 novembre 1902 (à 2 semaines), agence de Doullens.

23. DASES, Éphraïm Lebœuf, né 25 septembre 1902, admis $1^{\text {er }}$ décembre 1910 (à 8 ans), abandonné, agence d'Abbeville.

24. DASES, Lukas Hermann, né vers 1901, admis 9 septembre 1910 (à 9 ans), agence de Romorantin.

25. DASES, Ernest Freugnac, né 17 janvier 1902, admis 2 juin 1902 (à 4 mois), abandonné, agence de Romorantin.

26. DASES, Bernadette Godin, née 12 août 1902, admise 13 août 1902 (à 1 jour), agence de Doullens.

27. DASES, Bernadette Couffe, née 26 octobre 1903, admise 3 août 1904 (à 9 mois), abandonnée, agence de Romorantin; Marcel Charles, né 5 avril 1900, agence de Romorantin. 
l'anonymat des parents demeurait absolu et les recherches de filiation impossibles. À partir des années 1860, la généralisation de l'abandon à bureau ouvert, procédure au cours de laquelle la mère est invitée à décliner son identité (ce qu'elle fait le plus souvent), laisse augurer de possibles retrouvailles : le nom et l'adresse de la mère figurent dans le dossier de l'enfant. Tout au long de la Troisième République et jusqu'à la fin des années 1970, l'administration oppose toujours une fin de non-recevoir aux demandes des anciens pupilles, conformément à l'article 36 de la loi du 27 juin 1904 relatif au secret professionnel dans les services d'enfants assistés. Elle n'envisage une mise en relations que si les parents en font expressément la demande: dans ce cas, elle diligente une enquête et tranche en fonction de critères socio-économiques.

Pendant la majeure partie du $\mathrm{XX}^{\mathrm{e}}$ siècle, l'administration oppose aux demandes un refus systématique. La plupart du temps, elle se contente d'envoyer au requérant une lettre type dans laquelle elle affirme que le dossier contient peu d'informations, que celles-ci, dénuées d'intérêt, remontent à une époque ancienne et que, si la mère se faisait connaître, la mise en relation serait alors envisageable. En octobre 1923, depuis Saïgon où il sert comme soldat, un pupille demande à « connaître [sa] mère qu'[il n'a] jamais embrassée ${ }^{28}$. En décembre 1923, une jeune fille tout juste majeure se fait connaître à l'administration générale: «[Je voudrais obtenir] l'adresse de ma mère à qui je pardonne du plus profond de mon cœur de m'avoir déposée à l'Assistance publique car j'ai la certitude absolue que ma mère m'a déposée espérant qu'il viendrait des jours meilleurs pour me retirer ${ }^{29}$. En août 1924, à peine libéré du service militaire, un pupille majeur demande au directeur de l'Assistance publique l'adresse de « la femme qui [lui] a donné le jour ${ }^{30}$. À ces trois lettres, l'administration répond par un refus catégorique et non motivé.

La lutte des enfants assistés pour accéder à leurs origines est lente à se mettre en place. Dans un premier temps, les anciens pupilles les plus résolus n'ont d'autre choix que de rechercher des appuis haut placés. Déboutée par l'administration, une jeune fille majeure se tourne vers le Président de la République en novembre 1924. Après s'être excusée « d'oser [s]'adresser en termes aussi simples à [sa] Majesté », elle expose son cas en précisant qu'elle s'est déjà adressée en vain à la préfecture de police de la Seine. Sachant que le Président de la République est « le protecteur des faibles », elle demande des " conseils nécessaires à [lui] faire retrouver [ses] parents [sic]». La Présidence de la République fait suivre à l'administration générale de
l'Assistance publique à Paris, qui répète qu'elle ne peut délivrer aucune information. À l'automne 1934, l'ancienne pupille implore l'aide d'Alexis Danan. Le célèbre journaliste de Paris-Soir, accoutumé à s'attaquer aux forteresses bureaucratiques, écrit à l'administration générale, laquelle lui répond que les relations ne sont possibles que si les parents le souhaitent. En novembre 1951, âgée de 48 ans, l'ancienne pupille revient à la charge: «Il serait d'un grand intérêt pour moi d'avoir des indications sur mes origines ». Enfin, en juillet 1959, à la veille de ses 56 ans, elle demande à nouveau des renseignements sur sa famille : «Ce serait pour moi une grande joie de voir cette demande se réaliser. [...] L'affaire [ill.] parue dans tous les journaux m’a révélé que vous connaissiez les détails sur vos pupilles ${ }^{31}$. Cinq refus n'ont pas découragé cette femme, qui s'est adressée successivement à l'administration, au pouvoir politique et au pouvoir journalistique. Persuadée que l'Assistance publique en sait beaucoup plus que ce qu'elle veut bien admettre (ce qui est faux, en l'occurrence, ses parents l'ayant exposée), elle vieillit sans rien savoir de sa famille; et l'on devine que ce secret la ronge. Sa pugnacité montre tout de même qu'une évolution est à l'œuvre.

Dans les années 1970, des associations se constituent et se mobilisent pour faire valoir le droit d'accès des anciens pupilles à leur dossier et surtout demander la levée du secret de la filiation. C'est la principale revendication de Pierre Verdier, infatigable militant du droit de l'enfant à connaître ses origines ${ }^{32}$. Claude Sageot dénonce quant à lui la situation des «sans-papiers d'origine». Pour lui, les pupilles comme les mères sont dépossédés par la rupture des liens ; l'État organise la perte « des origines d'un individu, avec son cortège de déplacements, de mensonges, d'humiliations, de trahisons ${ }^{33}$. Ces arguments conduisent l'État à assouplir sa position. À la fin des années 1970, les règles sur la communication des dossiers sont modifiées : l'article 6 bis de la loi du 17 juillet 1978 donne au pupille le droit d'accéder à son propre dossier et l'article 7 de la loi du 3 janvier 1979 fixe des délais spéciaux pour la consultation des dossiers. Une décision du Conseil d'État en date du 10 janvier 1996 précise que les dossiers des pupilles sont librement communicables, sauf pièces particulières, après un délai de soixante ans.

Parallèlement, le rapport Bianco-Lamy de 1979, fondé sur des considérations budgétaires, révolutionne les pratiques en encourageant le maintien des enfants à domicile $^{34}$. La loi du 6 juin 1984 avance l'idée d'une collaboration entre les services sociaux et les familles : le consentement de ces dernières est obligatoire pour une prise

28. DASES, Michel Gredon, né 16 juin 1902, admis 27 février 1915 (à 12 ans et demi), en dépôt, agence de Romorantin.

29. DASES, Bérangère Mibord, née 22 décembre 1902, admise 24 décembre 1902 (à 2 jours), agence de Saint-Pol.

30. DASES, Victor Malèvre, né 28 septembre 1902, admis 9 octobre 1902 (à 11 jours), abandonné, agence d'Abbeville.

31. DASES, Jeannette Dalz, née 22 août 1903, admise 3 septembre 1903 (à 10 jours), trouvée, agence de Romorantin.

32. Voir P. Verdier, L'Enfant en miettes, Paris, Privat, 1978; P. Verdier, G. Delaisi, Enfant de personne, Paris, Odile Jacob, 1994; P. Verdier, M. Duboc, Face au secret des origines. Le droit d'accès au dossier des enfants abandonnés, Paris, Dunod, 1996. P. Verdier anime la Coordination des actions pour le droit à la connaissance des origines (CADCO), qui milite pour la suppression de l'accouchement sous X.

33. C. Sageot, Droit d'origine. La parole des acteurs, Paris, L'Harmattan - DPEAO, 1999, p. 8. Le DPEAO est une association qui milite pour le « droit des pupilles de l'État et des adoptés à leur origine». On mentionnera aussi le combat de G. Souty-Baum au sein du Mouvement national pour le droit d'accès aux origines familiales.

34. J.-L. Bianco, P. Lamy, L’Aide à l’enfance demain, Paris, Ministère de la Santé et de la Sécurité sociale (Études et Documents), 1980. 
en charge socio-éducative, le placement hors du domicile parental étant envisagé comme une mesure transitoire, et seulement si la sécurité de l'enfant l'impose. En cas de retrait de l'enfant, les relations avec les parents biologiques sont autorisées, les couples adoptants étant parfois euxmêmes favorables au contact avec la famille d'origine.

L'administration a beau assouplir les conditions d'accès aux dossiers, le secret de la filiation demeure une pomme de discorde entre l'État et les anciens pupilles. Ces derniers s'appuient sur la Convention internationale des droits de l'enfant signée en 1989, qui stipule que l'identité est un droit. En 1993, la Convention de La Haye a également prescrit que les États « veillent à conserver les informations qu'ils détiennent sur les origines de l'enfant et assurent l'accès de l'enfant à ces informations ». Précisément, les anciens pupilles ont l'impression que l'administration toute-puissante veut manipuler leur identité et effacer leur histoire, voire leur cacher des secrets. À ce sentiment de dépossession et d'injustice, l'État répond qu'il doit arbitrer entre deux volontés, celle de la mère et celle de l'enfant abandonné, et qu'il doit faire respecter les droits de deux vies privées.

Depuis la fin des années 1990, la situation a évolué vers un compromis. Ségolène Royal, ministre déléguée à l'Enfance et à la Famille dans le gouvernement de Lionel Jospin, a fait voter une loi relative à «l'accès aux origines personnelles». Pour elle, l'État doit faire en sorte "que tout enfant ait accès à son histoire ». La loi du 10 janvier 2002, texte de compromis, préserve la liberté de la femme et les secrets de famille (à la satisfaction des féministes et d'une partie de la droite), mais permet les retrouvailles (comme le demandaient les associations de pupilles) : «On peut, pour le respect de l'intégrité de l'enfant, reconnaître que plusieurs adultes tiennent un rôle dans sa vie, sans confusion des places, droits et responsabilités ${ }^{35}$. Selon la loi, l'accouchement sous X protège toujours l'identité de la mère; mais le secret de la filiation est désormais réversible. Au moment de la naissance, la mère qui accouche sous $\mathrm{X}$ est incitée à décliner son identité sous pli fermé. Si elle en est d'accord, son enfant pourra connaître plus tard son identité. Un Comité national de l'accès aux origines personnelles (CNAOP) est chargé de mettre en relation les enfants nés sous $\mathrm{X}$ et les mères désireuses de faire con- naître leur identité. Il reste que le droit français demeure l'un des plus restrictifs d'Europe. Pascale Odièvre, pupille de l'État née sous X en 1965 et adoptée en 1969, a porté devant la Cour européenne des droits de l'homme le litige qui l'oppose à l'État français : la levée du secret de sa filiation. En 2003, la Cour lui a donné tort ${ }^{36}$.

S'il est vrai que la famille, fondée sur la puissance maritale et paternelle telle qu'elle est définie par le Code civil, est au XIX ${ }^{\mathrm{e}}$ et au $\mathrm{XX}^{\mathrm{e}}$ siècle l'une des cellules fondamentales de la société, il apparaît que l'enfant abandonné est plus que tout autre un enfant dépourvu de lien social, un individu hors du droit. De 1811 à 2003, l'État-providence en charge des enfants - que son nom soit Assistance publique, Direction départementale de l'action sanitaire et sociale ou Aide sociale à l'enfance - a une conception de l'enfant cohérente et intangible : il prive l'enfant de certains droits tout en lui en garantissant d'autres.

Cette conception de la personne de l'enfant et de ses droits révèle une ambiguïté. Après avoir rompu les liens du sang, l'État assure à l'enfant, implanté dans son nouveau milieu, un certain nombre de droits : le droit à l'éducation familiale, le droit à la santé et à l'instruction, la défense des biens et des intérêts moraux. Historiquement, l'Assistance publique joue même un rôle pionnier en reconnaissant la personnalité juridique du mineur. Dans certains cas, la protection qu'elle garantit à l'enfant est meilleure que celle assurée aux enfants de famille : ainsi, les pupilles de l'Assistance publique ont bénéficié dès le milieu du XIX ${ }^{\mathrm{e}}$ siècle de la possibilité d'être scolarisés, hospitalisés ou embauchés par un contrat écrit. Il est vrai que ces droits restent parfois inappliqués, et l'affaire dite des «disparues de l'Yonne» révèle, à la fin du XX siècle, combien les pupilles de la DDASS restent inférieurs aux enfants de famille en matière de protection juridique. En 2000, le journal Le Monde écrivait dans son éditorial que ces jeunes filles ont été «doublement abandonnées, comme si, à l'injustice de leur naissance, on avait ajouté l'indifférence aveugle à leur sort de pauvres d'entre les pauvres $»{ }^{37}$.

Les droits que l'Assistance publique reconnaît au mineur reposent sur un principe : la rupture avec le milieu biologique, c'est-à-dire l'impossibilité de connaître ses

35. Entretien avec S. Royal dans Libération, 17 janvier 2001.

36. Dans l'arrêt Odièvre du 13 février 2003, la Cour a statué qu'il n'y avait pas eu violation de l'article 8 de la Convention européenne des droits de l'homme (droit à la vie privée et familiale) et qu'il n'y avait pas eu violation de l'article 14 de la Convention combiné avec l'article 8 (interdiction de la discrimination). Voir I. Marchand, D. Mendelsohn, De mère inconnue. Pascale Odièvre ou le combat des enfants nés sous X, Paris, Albin Michel, 2004.

37. Le Monde, 27 décembre 2000. Émile Louis, né à Auxerre en 1934 de parents inconnus, élevé par un maçon, marié à une nourrice de la DDASS et père de quatre enfants, conduisait dans les années 1970 et 1980 le bus qui faisait la navette entre des institutions spécialisées de l'Yonne et des familles d'accueil. Condamné à deux reprises pour des attouchements sur enfants, il est accusé en 2000 du viol et du meurtre de sept jeunes filles dans la région d'Auxerre. Plus de quinze jeunes femmes, pupilles de la DDASS, sans famille proche, légèrement handicapées et souvent pensionnaires de l'institut médico-éducatif Grattery d'Auxerre, ont disparu dans la région entre 1977 et 1989. À l'époque, leur « fugue » n'avait inquiété personne, hormis un gendarme ignoré de sa hiérarchie. Il a fallu que l'Association de défense des handicapées de l'Yonne (ADHY) porte plainte en 1996 pour que l'État prenne ses responsabilités et finisse, quatre ans plus tard, par mettre Émile Louis en examen. On a coutume d'associer ses crimes à deux autres affaires survenues dans l'Yonne à la même époque et dont les victimes sont également des jeunes filles en situation de grande vulnérabilité. Le 31 octobre 1991 , Claude et Monique Dunand ont été condamnés pour viols et tortures sur des jeunes filles de la DDASS, séquestrées dans un pavillon d'Appoigny, près d'Auxerre, et livrées à une clientèle fortunée. Enfin, Pierre Charrier, secrétaire général de l'Association pour adultes et jeunes handicapés (APAJH) de l'Yonne, a été accusé de viol sur une jeune fille handicapée de 23 ans et condamné en correctionnelle à six ans de prison le 18 mai 1992. Les éléments de ces trois affaires - violences et crimes sexuels commis sur des pupilles handicapées de l'Assistance publique, chape de silence maintenue avec la complicité des notables locaux, inertie des pouvoirs publics - rappellent les scandales de la fin du XIX $\mathrm{e}^{\mathrm{e}}$ siècle. 
origines. Cette culture du secret est l'un des traits distinctifs de l'Assistance publique à la française. Aujourd'hui encore, la France est le seul pays européen à organiser la destruction de la filiation et à interdire toute recherche lorsque la mère a requis l'anonymat. 